ISSN 1112-9867

\title{
KINETICS ANALYSIS OF STEP AND JUMP FORWARD LUNGE AMONG BADMINTON PLAYERS
}

\author{
A. M. Nadzalan*, N. I. Mohamad, J. F. L. Low, K. Tan, M. Janep and S. Hamzah \\ Faculty of Sports Science and Coaching, Universiti Pendidikan Sultan Idris, Tanjung Malim, \\ Perak, Malaysia
}

Published online: 10 November 2017

\begin{abstract}
This study was conducted to determine and compare the kinetics during step forward lunge (SFL) and jump forward lunge (JFL) in badminton. Fifteen university badminton players (mean age $=22.07 \pm 1.39$ years old) were recruited and were assigned to perform SFL and JFL while holding a badminton racquet using their dominant hand. For both dominant and non-dominant leg, all the force variables during JFL were significantly higher compared to SFL. Results also showed that time to peak force and stance time was significantly shorter during SFL compared to JFL. Besides that, all the forces variables were greater in the dominant limb compared to the non-dominant limb. Time to peak force and stance time were also shorter in dominant limb compared to the non-dominant limb. To conclude, coaches and athletes need to be aware of the mechanical demands during both lunge method and the assymetries that exist in terms of force production between both sites of limbs.
\end{abstract}

Keywords: concentric; eccentric; impact; force; asymmetry.

Author Correspondence, e-mail: ali.nadzalan@fsskj.upsi.edu.my

doi: http://dx.doi.org/10.4314/jfas.v9i6s.74 


\section{INTRODUCTION}

Badminton is an intermittent sport characterized by multiple intense actions [1] including fast accelerations, decelerations, and many explosive movements with changes of direction over short distances [2-5]. Among the movement that always been performed in a badminton game is lunge [6]. Lunge was performed in a game when the player wants to retrieve a drop shot to reach the shuttlecock. Sturgess and Newton [1] had highlighted the importance of the ability to accelerate from receiving stance to retrieving a drop shot. Athletes should accelerate quickly with the lunge to the shuttlecock because reaching the drop shot late will either result in an error or will enable the opponent to easily attack a poorly returned shot.

In order to reach the shuttlecock, the player could perform the lunge either by step or jump. Both methods of lunge were thought to be the main ways used in order for badminton players to reach the shuttlecock. The lunge need to be performed quick in both descend and ascend phase to ensure a player can get ready for the next shot or return by the opponent. To perform a quick movement, the body need to produce great force during the starting of descend and ascend phase while at the same time need to face more injury risks from big ground reaction force that were produced during the landing of leading legs at the front.

It is important for the badminton players to determine the better ways to perform the lunge as the better way will ensure players can perform well in whole match with less risks of unjury. One of the things important to measure during the movement is the kinetic produced. While kinetic is the study of force that cause motion, the knowledge on kinetic responses will give clear picture on the demands of a badminton player so that training program can be planned accordingly $[7,8]$. Coaches and players also need to be aware of the force production in both dominant and non-dominant sites in both lunge protocols because assymetries iscrease to probabilities of injuries.

Despite several previous studies had shown the force production during lunge $[6,9,10]$, until now, lack of research existed on kinetics data of both dominant and non-dominant limb in both main protocols of lunge movement. It is the aim of this study to determine and compare the kinetics during both dominant and non-dominant limb during step and jump forward lunge and also between dominant and non-dominant limb during both lunge protocols. 


\section{METHODOLOGY}

\subsection{Participants}

This study involved university male badminton players as study participants $(n=15)$. Participants recruited were the currently active university representatives in any badminton tournament organized by national level university sports council. All the participants selected were males aged between 20-25 years old based on their year of birth. Participants were screened prior to testing using PAR Q. Each participant had read and signed an informed consent for testing and training approved by Universiti Pendidikan Sultan Idris.

\subsection{Step and jump forward badminton-specific lunge}

Figure 1 (a) and (b) showed the step for SFL and JFL. Participants were instructed to stand with one of their hand (preferred) holding a badminton racquet, feet shoulder width apart. Participants lunged forward and must lower the thigh until be parallel with the ground, and then returned back to the starting position. Participants were needed to make a big step as during downward position, the knee should not extend beyond the toe. As to mimic the movement used in real badminton game situation, participant bent their trunk to $45^{\circ}$ forward. During descent movement, participants were required to act like in the badminton real situation in which the hand holding the racquet should be reaching a shuttlecock. Jump forward lunge were performed similar to the step forward lunge except participants need to explosively (jump) lunged forward and then explosively (jump) returned back also by jumping to the starting position. Participants were required to perform all the SFL and JFL for three trials consisting of three repetitions for each trial for both dominant and non-dominant lower limb.

(a)

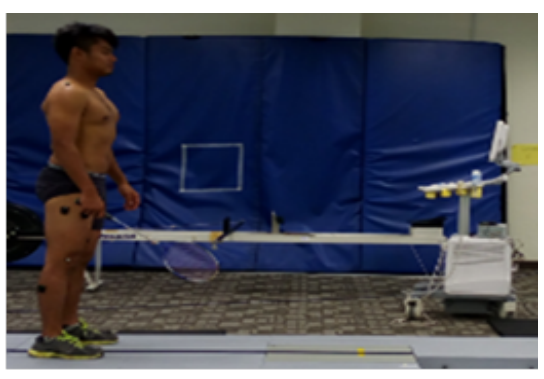

(b)

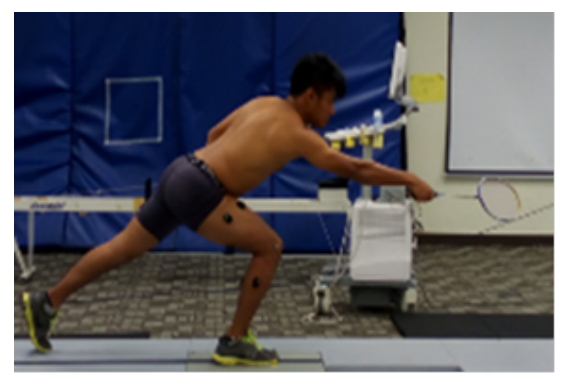

Fig.1. SFL and JFL performed in this study 


\subsection{Movement Kinetics}

Participants performed the lunge on a tri-axial force platform (BP400600HF-2000, AMTI Inc., USA) (width: $400 \mathrm{~mm} X$ length: $600 \mathrm{~mm}$ X height: $82.5 \mathrm{~mm}$ ). Data sampling rate were set at $200 \mathrm{~Hz}$ with filter cut-off frequency rate of $10 \mathrm{~Hz}$ [11]. The peak resultant GRF that was recorded from the force platform is defined as the magnitude of the resultant $\mathrm{X}, \mathrm{Y}$ and $\mathrm{Z}$ vectors.

The kinetics data that were measured in this study were the; i) absolute peak concentric force $(\mathrm{PCFa})$, ii) relative peak concentric force $(\mathrm{PCFr})$, iii) absolute mean concentric force (MCFa), iv) relative mean concentric force $(\mathrm{MCFr}), \mathrm{v})$ absolute mean eccentric force (MEFa), vi) relative mean eccentric force (MEFr), vii) absolute impact force (IFa), viii) relative impact force (IFr), ix) time to peak force (TPF) and x) stance time (ST). Peak concentric force was defined as the highest force before the takeoff. Mean concentric force was the average of force produced between the beginning of concentric phase and the end of concentric force. Mean eccentric force was the average of force produced between the start of participant step on the force platform until the point where the concentric force begin. The impact force is the first impact force that the participants start to step on the force platform. Time to peak force was the time taken between the beginnings of concentric force to the production of peak force during concentric phase. Stance time was the time taken from the start until the end time participants stepped on the force platform. The relative data refer to the all force data divided by bodyweight.

\subsection{Data Collection}

Prior to the test, all participants involved in a familiarization session in order to make sure all the participants were able to perform all the lunge movement correctly. In order to ensure maximal performance during the test, participants were instructed to "lunge as far as possible and as fast as possible". Movement kinetics of the stepping limb (dominant and non-dominant) was assessed during each test. Comparisons of those variables were made between each lunge protocols and between dominant and non-dominant limbs. All the lunge technique were closely monitored and controlled throughout all sessions. Participants were required to perform all exercises to a parallel depth as determined by the femoral line (line 
between the greater trochanter and the lateral epicondyle) being parallel to the ground. All the familiarization and data collection sessions were supervised by the researcher with the assistance of appointed trained trainers.

\subsection{Statistical Analysis}

Descriptive statistics were used to measure the mean and standard deviation of each physical characteristics and data scores. Repeated measure analysis of multivariances (MANOVA) was used to compare the difference of movement kinetics. Statistical significance was accepted at an $\alpha$-level of $\mathrm{p} \leq 0.05$. All statistical analyses were conducted using SPSS version 23 (IBM, New York, USA).

\section{RESULTS}

\subsection{Physical Characteristics}

Table 1 showed the physical characteristics of participants involved in this study.

Table 1. Physical Characteristics of Participants

\begin{tabular}{ll}
\hline Variables & Mean $\pm \mathrm{SD}$ \\
\hline Age (years) & $22.07 \pm 1.39$ \\
Body Mass $(\mathrm{kg})$ & $70.07 \pm 1.88$ \\
Body Weight $(\mathrm{N})$ & $687.41 \pm 13.53$ \\
Height $(\mathrm{cm})$ & $173.13 \pm 2.12$ \\
1RM $(\mathrm{kg})$ & $71.87 \pm 2.59$ \\
Relative $1 \mathrm{RM}(1 \mathrm{RM} / \mathrm{BM})$ & $1.03 \pm 0.01$ \\
\hline
\end{tabular}

\subsection{Dominant Lower Limb}

Analysis of the dominant lower limb showed significant main effect for all the kinetic variables: i) absolute peak concentric force $(\mathrm{PCFa}), \mathrm{F}(1,14)=26616.588 ; \mathrm{p}<0.001$, ii) relative peak concentric force $(\mathrm{PCFr}), \mathrm{F}(1,14)=103757.743 ; \mathrm{p}<0.001$, iii) absolute mean concentric force $(\mathrm{MCFa}), \mathrm{F}(1,14)=28460.431 ; \mathrm{p}<0.001$, iv) relative mean concentric force $(\mathrm{MCFr}), \mathrm{F}(1,14)=16177.331 ; \mathrm{p}<0.001, \mathrm{v})$ absolute mean eccentric force $(\mathrm{MEFa}), \mathrm{F}(1,14)=$ 22591.352; $\mathrm{p}<0.001$, vi) relative mean eccentric force $(\mathrm{MEFr}), \mathrm{F}(1,14)=71034.851 ; \mathrm{p}<$ 
0.001 , vii) absolute impact force (IMPFa), $\mathrm{F}(1,14)=5859.225 ; \mathrm{p}<0.001$, viii) relative impact force $(\mathrm{IMPFr}), \mathrm{F}(1,14)=10912.046 ; \mathrm{p}<0.001$, ix) time to peak force $(\mathrm{TPF}), \mathrm{F}(1,14)=$ 173.103; $\mathrm{p}<0.001$ and $\mathrm{x}$ ) stance time (ST), $\mathrm{F}(1,14)=436.407 ; \mathrm{p}<0.001$.

Table 2. Kinetics Data of Dominant Limb during SFL and JFL

\begin{tabular}{cll}
\hline Kinetics & SFL & JFL \\
\hline PCFa & $745.92 \pm 19.74^{\mathrm{b}}$ & $1071.68 \pm 27.05^{\mathrm{a}}$ \\
PCFr & $1.09 \pm 0.98^{\mathrm{b}}$ & $1.56 \pm 0.83^{\mathrm{a}}$ \\
MCFa & $659.77 \pm 25.98^{\mathrm{b}}$ & $967.67 \pm 25.43^{\mathrm{a}}$ \\
MCFr & $0.96 \pm 0.02^{\mathrm{b}}$ & $1.41 \pm 0.01^{\mathrm{a}}$ \\
MEFa & $536.49 \pm 15.29^{\mathrm{b}}$ & $867.33 \pm 22.46^{\mathrm{a}}$ \\
MEFr & $0.78 \pm 0.01^{\mathrm{b}}$ & $1.26 \pm 0.01^{\mathrm{a}}$ \\
IMPFa & $764.53 \pm 19.28^{\mathrm{b}}$ & $1235.42 \pm 35.86^{\mathrm{a}}$ \\
IMPFr & $1.11 \pm 0.02^{\mathrm{b}}$ & $1.80 \pm 0.02^{\mathrm{a}}$ \\
TPF & $0.43 \pm 0.01^{\mathrm{b}}$ & $0.51 \pm 0.03^{\mathrm{a}}$ \\
ST & $0.93 \pm 0.04^{\mathrm{b}}$ & $1.04 \pm 0.03^{\mathrm{a}}$ \\
\hline & $\mathrm{a}=$ significantly difference from SFL, $\mathrm{p}<0.001, \mathrm{p}<0.01, \mathrm{p}<0.05$ \\
b $=$ significantly difference from JFL, $\mathrm{p}<0.001, \mathrm{p}<0.01, \mathrm{p}<0.05$
\end{tabular}

Table 2 showed the kinetics data during the two lunge protocols. Pairwise comparison showed that all the force variables (PCFa, PCFr, MCFa, MCFr, MEFa, MEFr, IMPFa and IMPFr) during JFL were significantly higher compared to SFL, $\mathrm{p}<0.001$. Results also showed that time to peak force and stance time was significantly shorter during SFL compared to JFL, $\mathrm{p}<$ 0.001 .

\subsection{Non-dominant Lower Limb}

Analysis of the non-dominant lower limb showed significant main effect were found for all the kinetic variables: i) absolute peak concentric force (PCFa), $\mathrm{F}(1,14)=19342.012 ; \mathrm{p}<$ 0.001 , ii) relative peak concentric force $(\mathrm{PCFr}), \mathrm{F}(1,14)=86723.410 ; \mathrm{p}<0.001$, iii) absolute mean concentric force $(\mathrm{MCFa}), \mathrm{F}(1,14)=26723.214 ; \mathrm{p}<0.001$, iv) relative mean concentric 
force $(\mathrm{MCFr}), \mathrm{F}(1,14)=14234.82 ; \mathrm{p}<0.001, \mathrm{v})$ absolute mean eccentric force $(\mathrm{MEFa})$, $\mathrm{F}(1,14)=22591.352 ; \mathrm{p}<0.001$, vi) relative mean eccentric force $(\mathrm{MEFr}), \mathrm{F}(1,14)=$ 68231.02; $\mathrm{p}<0.001$, vii) absolute impact force (IMPFa), $\mathrm{F}(1,14)=6232.218 ; \mathrm{p}<0.001$, viii) relative impact force (IMPFr), $\mathrm{F}(1,14)=8780.120 ; \mathrm{p}<0.001$, ix) time to peak force (TPF), $\mathrm{F}(1,14)=110.234 ; \mathrm{p}<0.001$ and $\mathrm{x})$ stance time $(\mathrm{ST}), \mathrm{F}(1,14)=346.230 ; \mathrm{p}<0.001$.

Table 3. Kinetics Data of Non-Dominant Limb during SFL and JFL

\begin{tabular}{cll}
\hline Kinetics & SFL & JFL \\
\cline { 2 - 3 } PCFa & $715.91 \pm 21.05)^{\mathrm{b}}$ & $1006.68 \pm 31.03^{\mathrm{a}}$ \\
PCFr & $1.04 \pm 0.02)^{\mathrm{b}}$ & $1.46 \pm 0.03^{\mathrm{a}}$ \\
MCFa & $634.77 \pm 27.27)^{\mathrm{b}}$ & $932.67 \pm 26.85^{\mathrm{a}}$ \\
MCFr & $0.92 \pm 0.02)^{\mathrm{b}}$ & $1.36 \pm 0.02^{\mathrm{a}}$ \\
MEFa & $512.15 \pm 17.68)^{\mathrm{b}}$ & $830.66 \pm 24.15^{\mathrm{a}}$ \\
MEFr & $0.75 \pm 0.02)^{\mathrm{b}}$ & $1.21 \pm 0.02^{\mathrm{a}}$ \\
IMPFa & $736.20 \pm 19.53)^{\mathrm{b}}$ & $1191.09 \pm 36.20^{\mathrm{a}}$ \\
IMPFr & $1.07 \pm 0.02)^{\mathrm{b}}$ & $1.73 \pm 0.02^{\mathrm{a}}$ \\
TPF & $0.46 \pm 0.01)^{\mathrm{b}}$ & $0.55 \pm 0.03^{\mathrm{a}}$ \\
ST & $0.95 \pm 0.04)^{\mathrm{b}}$ & $1.07 \pm 0.03^{\mathrm{a}}$ \\
\hline a $=$ significantly difference from SFL $, \mathrm{p}<0.001, \mathrm{p}<0.01, \mathrm{p}<0.05$ \\
b $=$ significantly difference from JFL $, \mathrm{p}<0.001, \mathrm{p}<0.01, \mathrm{p}<0.05$
\end{tabular}

Table 3 showed the kinetics data during the two lunge protocols. As in dominant limb, pairwise comparison test showed that all the force variables (PCFa, PCFr, MCFa, MCFr, MEFa, MEFr, IMPFa and IMPFr) during JFL were significantly higher compared to SFL, $p<$ 0.001. Results also showed that time to peak force and stance time was significantly shorter during SFL compared to JFL, $\mathrm{p}<0.001$.

\subsection{Step Forward Lunge (Dominant versus Non-dominant Lower Limb)}

Analysis of the dominant and non-dominant lower limb during step forward lunge showed significant main effect were found for all the kinetic variables: i) absolute peak concentric 
force $(\mathrm{PCFa}), \mathrm{F}(1,14)=189.00 ; \mathrm{p}<0.001$, ii) relative peak concentric force $(\mathrm{PCFr}), \mathrm{F}(1,14)=$ 190.298; $\mathrm{p}<0.001$, iii) absolute mean concentric force $(\mathrm{MCFa}), \mathrm{F}(1,14)=131.250 ; \mathrm{p}<$ 0.001 , iv) relative mean concentric force $(\mathrm{MCFr}), \mathrm{F}(1,14)=132.174 ; \mathrm{p}<0.001$, v) absolute mean eccentric force $(\mathrm{MEFa}), \mathrm{F}(1,14)=93.962 ; \mathrm{p}<0.001$, vi) relative mean eccentric force (MEFr), $\mathrm{F}(1,14)=94.634 ; \mathrm{p}<0.001$, vii) absolute impact force (IMPFa), $\mathrm{F}(1,14)=289.00 ; \mathrm{p}$ $<0.001$, viii) relative impact force (IMPFr), $\mathrm{F}(1,14)=289.00 ; \mathrm{p}<0.001$, ix) time to peak force $(\mathrm{TPF}), \mathrm{F}(1,14)=189.00 ; \mathrm{p}<0.001$ and $\mathrm{x}$ ) stance time $(\mathrm{ST}), \mathrm{F}(1,14)=189.00 ; \mathrm{p}<$ 0.001 .

Pairwise comparison test showed that all the forces variables were greater in the dominant limb compared to the non-dominant limb. Besides that, time to peak force and stance time were also shorter in dominant limb compared to the non-dominant limb.

\subsection{Jump Forward Lunge (Dominant versus Non-dominant Lower Limb)}

Analysis of the dominant and non-dominant lower limb during jump forward lunge showed significant main effect were found for all the kinetic variables: i) absolute peak concentric force $(\mathrm{PCFa}), \mathrm{F}(1,14)=221.813 ; \mathrm{p}<0.001$, ii) relative peak concentric force $(\mathrm{PCFr}), \mathrm{F}(1,14)$ $=223.270 ; \mathrm{p}<0.001$, iii) absolute mean concentric force $(\mathrm{MCFa}), \mathrm{F}(1,14)=257.250 ; \mathrm{p}<$ 0.001 , iv) relative mean concentric force $(\mathrm{MCFr}), \mathrm{F}(1,14)=258.827 ; \mathrm{p}<0.001$, v) absolute mean eccentric force $(\mathrm{MEFa}), \mathrm{F}(1,14)=255.891 ; \mathrm{p}<0.001$, vi) relative mean eccentric force (MEFr), $\mathrm{F}(1,14)=257.292 ; \mathrm{p}<0.001$, vii) absolute impact force (IMPFa), $\mathrm{F}(1,14)=555.260$; $\mathrm{p}<0.001$, viii) relative impact force (IMPFr), $\mathrm{F}(1,14)=551.875 ; \mathrm{p}<0.001$, ix) time to peak force $(\mathrm{TPF}), \mathrm{F}(1,14)=223.155 ; \mathrm{p}<0.001$ and $\mathrm{x}$ ) stance time $(\mathrm{ST}), \mathrm{F}(1,14)=131.042 ; \mathrm{p}<$ 0.001 .

As during SFL, pairwise comparison test showed that all the forces variables were greater in the dominant limb compared to the non-dominant limb. Besides that, time to peak force and stance time were also shorter in dominant limb compared to the non-dominant limb.

\section{DISCUSSIONS}

Absolute peak concentric force (PCFa), relative peak concentric force (PCFr), absolute mean concentric force $(\mathrm{MCF})$, relative mean concentric force $(\mathrm{MCFr})$, absolute mean eccentric 
force (MEFa), relative mean eccentric force (MEFr), absolute impact force (IMPFa), relative impact force (IMPFr), time to peak force (TPF), and stance time (ST) were assessed and compared between each lunge protocols in this study.

Results showed that all the force variables (PCFa, PCFr, MCFa, MCFr, MEFa, MEFr, IMPFa and IMPFr) during JFL were significantly higher compared to SFL, $\mathrm{p}<0.001$. Results also showed that time to peak force and stance time was significantly shorter during SFL compared to JFL, $\mathrm{p}<0.001$. These conditions were observed in both dominant and non-dominant limbs.

Results demonstrated that performing lunge with jumping will produce greater force production during eccentric and concentric phase. JFL was thought to produce greater force due to the explosiveness that was needed in order to produce the movement especially during concentric phase in which participant need to produce great force in order for them to jump back into starting position [12]. Eccentric force was found to be higher might be due to the impact force during landing on the force platform [13]. The jumping movement cause participants to lift their lower limb higher before landing thus cause the impact to be greater. Badminton players need to place a greater attention to the greater impact and eccentric force produced during JFL because this might place more stress to their lower limb joint and muscle thus the inability to control the movement during descent phase might induces greater injury risks [14].

Performing a movement in jumping manner were shown to be more effective in force production and was in line with previous study of badminton lunge [10]. Larger peak vertical force during JFL could be related to the generation of extensor force by the knee joint to lift the leg off the ground for the jump phase prior to the secondary ground contact [10]. Several previous studies have found the advantageous of performing the lunge with jump movements $[10,15]$. The application of larger forces horizontally and vertically at the secondary ground contact of the hop lunge would allow the participants to generate greater speed at drive-off and thus would enable the participants to recover more quickly [10, 15].

Although the stance time was showed to be significantly longer, the total task duration was only slightly and not-significantly longer for the JFL compared to SFL. This showed that different protocols of lunge performed could cause a minor influence on the task performance. 
Quicker recovery phase of the hop lunge compensated for the longer stance phase, and the faster recovery may indeed be advantageous in a situation where it is necessary to carry the momentum on beyond the start position to respond to, for example, a clearing shot played to the back of the court [10]. Although no data of recovery phase was determined in this current study, when looking at the previous findings, it should be noted that using the JFL would be more preferable compared to the SFL as this could result in the player to arrive at the shuttle faster especially when there is necessary for continued movement beyond the start position. Despite the force production data that obtained in this study, it was suggested that future studies to include power production during each lunge protocols. This is as a way to find the optimal loadings for power production in lunge exercise. The meta analysis conducted by Soriano, Jiménez-Reyes [16] demonstrated the differences in optimal load between the squat, jump squat, power clean, and hang power clean exercise. The intensity for optimal power production for each exercise was found to be different. For example, heavier loads $(70 \%$ $1 \mathrm{RM}$ ) were needed to optimize peak power production during power cleans and hang power cleans. This condition reflects the greater power production was perhaps due to the force production components. Despite of that, lighter loads $(0-30 \%$ of $1 \mathrm{RM})$ were found to be better in optimizing peak power output during squat jump compared to moderate and heavy loads, thus showed that peak power production during squat jump were more affected by the ballistic nature of the movement [16].

It is currently lack of data to find out the optimal loadings to be used in lunge exercise. Future studies need to be conducted on this so that more information available for the selection of loads during different kind of exercises. Based on the meta analysis [16], lighter loads (0-30\%1RM) might be the optimal loads for peak power production during JFL as this might result in selective motor unit recruitment, increased firing frequency, and synchronization of active motor units targeting the early phase rate of force development [17-19]. However, no speculation can be made for traditional lunge movement (step forward lunge) as the movement is different from those that have been studies.

Comparing the dominant and non-dominant site, it was found that all the forces output were greater in the dominant site besides shorter stance time during both lunge protocols. The higher kinetics data in dominant site was contributed by the faster movement and more 
muscle activation produced that has been shown earlier. These again showed the importance of non-dominant site training to avoid strength imbalances that can negatively affect performance besides increase injury risk. The overall dominant and non-dominant site comparison demonstrate the existed of imbalances between the two sites as non-dominant were shown to be slower, and produces significantly lesser muscle activation and force production.

This current findings for kinetic data was in line to the previous findings of Hsieh, Huang [20] that found significant differences to be exist between dominant and non-dominant limb in kinetics variables during $360^{\circ}$ turning roundhouse kick. However, this is in contrast to the findings of Niu, Wang [21] that found no differences of kinetic variables during drop landing. Similar to Niu, Wang [21], Van der Harst, Gokeler [22] in their study also did not found any kinetic differences between dominant and non-dominant site during landing from a single-leg hop. The differences of findings in this study could be due to the differences of movement executed and participants recruited for the study. No comparison could be made to lunge movement as lack of study has been found on the differences of dominant and non-dominant lower limb kinetics during lunge.

\section{CONCLUSIONS}

Throughout the consistency of lunge used in sports, lunge exercises should be used widely as training exercises during strength training program. The inclusion of lunge as training exercises should be beneficial as it will allow athletes or individuals to train and improve their ability for the movement and as a way to overload the athletes or individuals, various methods of lunge could be implemented during training sessions [23]. This includes putting some weights and includes ballistic movement during the exercise.

\section{ACKNOWLEDGEMENTS}

List here those individuals who provided help during the research (e.g., providing language help, writing assistance or proof reading the article, etc.). 


\section{REFERENCES}

[1] Sturgess, S. and R.U. Newton, Design and implementation of a specific strength program for badminton. Strength \& Conditioning Journal, 2008. 30(3): p. 33-41.

[2] Chin, M., et al., Physiological profiles and sport specific fitness of Asian elite squash players. British Journal of Sports Medicine, 1995. 29(3): p. 158-164.

[3] Chin, M., et al., Sport specific fitness testing of elite badminton players. British Journal of Sports Medicine, 1995. 29(3): p. 153-157.

[4] Baker, D., Improving vertical jump performance through general, special, and specific strength training: A brief review. The Journal of Strength \& Conditioning Research, 1996. 10(2): p. 131-136.

[5] Hughes, M. and G. Bopf, Relationships between performance in jump tests and speed tests in elite Badminton players. Journal of Sports Sciences, 2005. 23: p. 194-195.

[6] Farrokhi, S., et al., Trunk position influences the kinematics, kinetics, and muscle activity of the lead lower extremity during the forward lunge exercise. Journal of Orthopaedic \& Sports Physical Therapy, 2008. 38(7): p. 403-409.

[7] Nadzalan, A.M., et al., The effects of step versus jump forward lunge exercise training on muscle architecture among recreational badminton players. World Applied Sciences Journal, 2017. 35(8): p. 1581-1587.

[8] Nadzalan, A.M., et al., Muscle activation analysis of step and jump forward lunge among badminton players. Journal of Engineering and Science Research, 2017. 1(2): p. 60-65.

[9] Riemann, B.L., et al., Biomechanical comparison of forward and lateral lunges at varying step lengths. The Journal of Sports Medicine and Physical Fitness, 2013. 53(2): p. 130-138.

[10]Kuntze, G., N. Mansfield, and W. Sellers, A biomechanical analysis of common lunge tasks in badminton. Journal of Sports Sciences, 2010. 28(2): p. 183-191.

[11]Mohamad, N.I., J.B. Cronin, and K.K. Nosaka, Difference in kinematics and kinetics between high-and low-velocity resistance loading equated by volume: Implications for hypertrophy training. The Journal of Strength \& Conditioning Research, 2012. 26(1): p. 269-275.

[12]Hakkinen, K., P. Komi, and M. Alen, Effect of explosive type strength training on isometric force - and relaxation - time, electromyographic and muscle fibre characteristics of 
leg extensor muscles. Acta Physiologica Scandinavica, 1985. 125(4): p. 587-600.

[13]McKay, H., et al., Ground reaction forces associated with an effective elementary school based jumping intervention. British Journal of Sports Medicine, 2005. 39(1): p. 10-14.

[14]Dickin, D.C., et al., Combined Effects of Drop Height and Fatigue on Landing Mechanics in Active Females. Journal of Applied Biomechanics, 2015. 31(4).

[15]Fukashiro, S., et al., In vivo achilles tendon loading'during jumping in humans. European Journal of Applied Physiology and Occupational Physiology, 1995. 71(5): p. 453-458.

[16]Soriano, M.A., et al., The optimal load for maximal power production during lower-body resistance exercises: a meta-analysis. Sports Medicine, 2015. 45(8): p. 1191-1205.

[17]Cormie, P., J.M. McBride, and G.O. McCaulley, Power-time, force-time, and velocity-time curve analysis during the jump squat: impact of load. Journal of Applied Biomechanics, 2008. 24(2): p. 112.

[18]Duchateau, J. and K. Hainaut, Electrical and mechanical failures during sustained and intermittent contractions in humans. Journal of Applied Physiology, 1985. 58(3): p. 942-947.

[19]Cutsem, M., J. Duchateau, and K. Hainaut, Changes in single motor unit behaviour contribute to the increase in contraction speed after dynamic training in humans. The Journal of Physiology, 1998. 513(1): p. 295-305.

[20]Hsieh, A., C.-F. Huang, and C.C. Huang. The biomechanical analysis of roundhouse kick in taekwondo. in ISBS-Conference Proceedings Archive. 2012.

[21]Niu, W., et al., Kinematics, kinetics, and electromyogram of ankle during drop landing: A comparison between dominant and non-dominant limb. Human Movement Science, 2011. 30(3): p. 614-623.

[22]Van der Harst, J., A. Gokeler, and A. Hof, Leg kinematics and kinetics in landing from a single-leg hop for distance. A comparison between dominant and non-dominant leg. Clinical Biomechanics, 2007. 22(6): p. 674-680.

[23]Baechle, T.R. and R.W. Earle, Essentials of strength training and conditioning. Vol. 7. 2008: Human Kinetics Champaign, IL.

\section{How to cite this article:}

Nadzalan A. M. Mohamad N. I., Low J. F. L., Tan K., Janep M., and Hamzah S. Kinetics Analysis of Step and Jump Forward Lunge among Badminton Players. J. Fundam. Appl. Sci., 2017, 9(6S), 1011-1023. 